\title{
THINKLET: ELEMENTO CLAVE EN LA GENERACIÓN DE MÉTODOS COLABORATIVOS PARA EVALUAR USABILIDAD DE SOFTWARE
}

\author{
THINKLET: KEY ELEMENT IN THE COLLABORATIVE METHODS GENERATION \\ FOR EVALUATE SOFTWARE USABILITY
}

\author{
Andrés Solano Alegría \\ Ing. de Sistemas, candidato a Magíster en Computación, Integrante del grupo IDIS \\ (Investigación y Desarrollo en Ingeniería de Software), Popayán, Colombia, \\ afsolano@unicauca.edu.co \\ Yenny Méndez Alegría \\ Ing. de Sistemas y Licenciada en Educación con Especialidad en Matemáticas, \\ candidata a Magíster en Computación, Integrante del grupo IDIS (Investigación \\ y Desarrollo en Ingeniería de Software), Popayán, Colombia, \\ ymendal@unicauca.edu.co \\ César Collazos Ordóñez \\ Ing. de Sistemas, Ph. D., Profesor titular de la Universidad del Cauca, Coordinador del grupo IDIS \\ (Investigación y Desarrollo en Ingeniería de Software), Popayán, Colombia, \\ ccollazo@unicauca.edu.co
}

Fecha de recepción: 2 de noviembre de 2011

Fecha de aprobación: 4 de diciembre de 2010

\section{RESUMEN}

En la actualidad, la usabilidad es un atributo fundamental para el éxito de un producto software. La competitividad entre organizaciones obliga a mejorar el nivel de usabilidad de los productos, debido al riesgo que existe de perder clientes, si el producto no es fácil de usar y/o fácil de aprender. Aunque se han establecido métodos para evaluar la usabilidad de productos software, la mayoría de estos métodos no consideran la posibilidad de involucrar a varias personas trabajando de forma colaborativa en el proceso de evaluación. Por esta razón, convendría utilizar la Metodología para el Diseño de Métodos de Evaluación de Usabilidad Colaborativos, de tal forma que se diseñen métodos que permitan a varias personas de diversas áreas de conocimiento, trabajar de forma colaborativa en el proceso de evaluación. Este artículo presenta de forma general, la metodología mencionada y hace especial énfasis en los thinklets, como elementos clave para el diseño de procesos colaborativos. 
Palabras Clave: usabilidad, métodos de evaluación de usabilidad, trabajo colaborativo, patrón de colaboración, thinklet.

\section{ABSTRACT}

Currently, usability is a critical attribute to success of software. The competition among organizations forces to improve the level of product usability due to the risk of losing customers if product would not be easy to use and/or easy to learn. Methods have been established to evaluate the usability of software products; however, most of these methods don't take into account the possibility to involve several people working collaboratively in the evaluation process. Therefore, Methodology for Design of Collaborative Usability Evaluation Methods should be used to design methods that allow several people from a range of knowledge areas to work collaboratively in the evaluation process. This paper presents the methodology mentioned and gives special emphasis on Thinklets, as key elements for design of collaborative processes.

Key Words: usability, usability evaluation method, collaborative work, collaboration pattern, thinklet.

\section{INTRODUCCIÓN}

En muchas organizaciones, el proceso de desarrollo de software incluye personas de diversas áreas de conocimiento. De igual forma, en el proceso de evaluación de la usabilidad de productos software, convendría disponer de un grupo de evaluadores interdisciplinarios, por ejemplo: expertos en usabilidad y diseño centrado en el usuario, arquitectos de información, diseñadores gráficos, psicólogos, etc., ya que realizar la evaluación de usabilidad es muy difícil sin la colaboración de otras personas, debido a que constantemente se encuentran actividades más complejas que requieren la experiencia de diversas personas. Además, la colaboración "permite coordinar toda una serie de actividades que apoyan la comunicación, la coordinación y la negociación entre varias personas, con el fin de aumentar la productividad" [1], y de esta manera, se tiende a maximizar los resultados y minimizar la pérdida de tiempo e información en el proceso de evaluación.

Aunque se han establecido métodos para evaluar la usabilidad de software, el inconveniente radica en que un gran número de ellos, se han diseñado para ser utilizados en un ambiente de trabajo individual. Desde esta perspectiva, este artículo presenta de forma general la aplicación de la Metodología para el Diseño de Métodos de Evaluación de Usabilidad Colaborativos, tomando como caso de estudio la generación del método colaborativo Entrevista, el cual permite que varias personas se involucren en el proceso de evaluación, con el objetivo de que los resultados de la evaluación 
sean más adecuados y se contribuya significativamente en el desarrollo de productos software usables. En el contenido del artículo, se destacan los thinklets como elementos clave en el diseño de procesos colaborativos, por que se pueden usar y adaptarse con facilidad para construir este tipo de procesos, inmersos en la evaluación de usabilidad de software.

En la siguiente sección, se presentan algunos referentes teóricos relacionados con la temática del artículo; luego, se presenta de forma general información sobre la Metodología para el Diseño de Métodos de Evaluación de Usabilidad Colaborativos y los resultados obtenidos en la ejecución de cada una de sus fases para el método de la Entrevista. Posteriormente, se presentan algunos thinklets generados mediante el desarrollo de la metodología, y por último, se presentan conclusiones y trabajo futuro.

\section{REFERENTES TEÓRICOS}

\subsection{USABILIDAD Y MÉTODOS PARA SU EVALUACIÓN}

La usabilidad se define como "el grado en el que un producto puede ser utilizado por usuarios específicos para conseguir objetivos específicos con efectividad, eficiencia y satisfacción en un determinado contexto de uso" [2]. La usabilidad es vista por lo general, para asegurar que los productos interactivos sean fáciles de aprender, efectivos y agradables para sus usuarios. Al realizar la tarea para la cual se ha desarrollado un software de manera intuitiva, eficiente y fácil, se puede pensar que el uso de este software será más fácil. La facilidad en el desarrollo de una tarea, se puede medir, verificando la rapidez con la cual se realiza, el número de errores cometidos y el grado de satisfacción del usuario al utilizar el software [3].

Existen muchos métodos de evaluación de usabilidad y algunos de ellos, parten de que el sistema ya está hecho o al menos hay un prototipo viable. Estos métodos se pueden "clasificar en diferentes categorías, tales como: inspección, indagación y prueba" [4]. La ejecución de estos métodos permite introducir mejoras en la usabilidad de un producto software, prototipo o versión definitiva de un sistema interactivo. Además, se realiza con el fin de detectar fallos o aspectos susceptibles de mejorar en cuanto a su uso.

\subsection{TRABAJO COLABORATIVO}

En décadas recientes, se han dado diferentes definiciones relacionadas con el trabajo colaborativo. Entre ellas se destacan las siguientes: Marx, en 1884, define el término trabajo colaborativo como: "múltiples individuos trabajando juntos de una manera 
planificada en un mismo proceso de producción o en procesos de producción diferentes pero conectados" [5]. De manera similar, Bannon define el trabajo colaborativo como "la nominación general y neutral de múltiples personas que trabajan juntas para producir un producto o servicio" [6]. El trabajo colaborativo persigue "el desarrollo de conocimiento compartido, la aceleración de los flujos de información, la coordinación de los flujos de recursos para producir economías de costos y tiempos" [7].

El aspecto más importante de trabajar en grupo, es el hecho de contar con un objetivo común que canaliza los esfuerzos individuales y ofrece un sentido de pertenencia que fomenta la unión. Los miembros del grupo tienen la oportunidad de aprender, tomando en consideración otros puntos de vista, maneras distintas de hacer las cosas, interpretaciones diferentes de conceptos, experiencia de otros y la forma como dan solución a problemas similares [8].

\subsection{PATRONES DE COLABORACIÓN}

Aunque se conozcan los pasos que un grupo de personas debe seguir para llevar a cabo un proceso y se tenga un conocimiento general de los resultados obtenidos durante la ejecución de cada paso, no se conoce necesariamente cómo el grupo ejecutará cada uno de ellos. De esta manera, los patrones de colaboración son una guía del cómo se ejecutará el proceso, y "definen la manera como los participantes de una actividad grupal van de un estado inicial a un estado final" [9].

\subsection{THINKLETS}

Los thinklets son bloques de construcción que se pueden unir para especificar cómo un determinado patrón de colaboración debe realizarse cuando el proceso está en ejecución. Un thinklet "constituye la unidad más pequeña del capital intelectual necesario para crear un patrón de la colaboración repetible y predecible, entre las personas que trabajan hacia un objetivo" [10].

La documentación que provee un thinklet, cuenta con la siguiente información [11]: un nombre metafórico o representativo relacionado con los patrones que crea el thinklet, criterios para decidir cuándo escoger o no el thinklet, información general del thinklet (como entradas y salidas), cómo usar el thinklet (pasos que conforman el thinklet), una historia exitosa que ayuda a clarificar las circunstancias bajo las cuales el Thinklet es útil y una explicación del nombre, la cual hace más fácil recordar el nombre del thinklet. 


\section{INCORPORACIÓN DE TRABAJO COLABORATIVO A LOS MÉTODOS DE EVALUACIÓN DE USABILIDAD}

\subsection{METODOLOGÍA PARA EL DISEÑO DE MÉTODOS DE EVALUACIÓN DE USABILIDAD COLABORATIVOS}

En los procesos que conforman los métodos de evaluación de usabilidad, el trabajo colaborativo se incorpora mediante la aplicación de la Metodología para el Diseño de Métodos de Evaluación de Usabilidad Colaborativos (figura 2) [12]. Esta metodología se obtuvo a partir de la Metodología para el Desarrollo de Procesos Colaborativos (figura 1) [13], planteada por investigadores de Ingeniería de Colaboración.

La Metodología para el Desarrollo de Procesos Colaborativos (metodología original propuesta por los investigadores), se compone de las siguientes fases [13]: Diagnóstico de la tarea, Evaluación de la actividad, Descomposición de la actividad, Relación de thinklets, Documentación de diseño y Validación del diseño (figura 1). Las fases de la metodología sufrieron algunas modificaciones, de tal manera que su uso permite generar métodos colaborativos para evaluar la usabilidad de software, es decir, métodos que permitan compartir conocimiento, recursos, etc., a varias personas durante su ejecución. La metodología propuesta, presenta modificaciones en la primera fase, entradas y salidas de las fases y algunas fases adicionales.

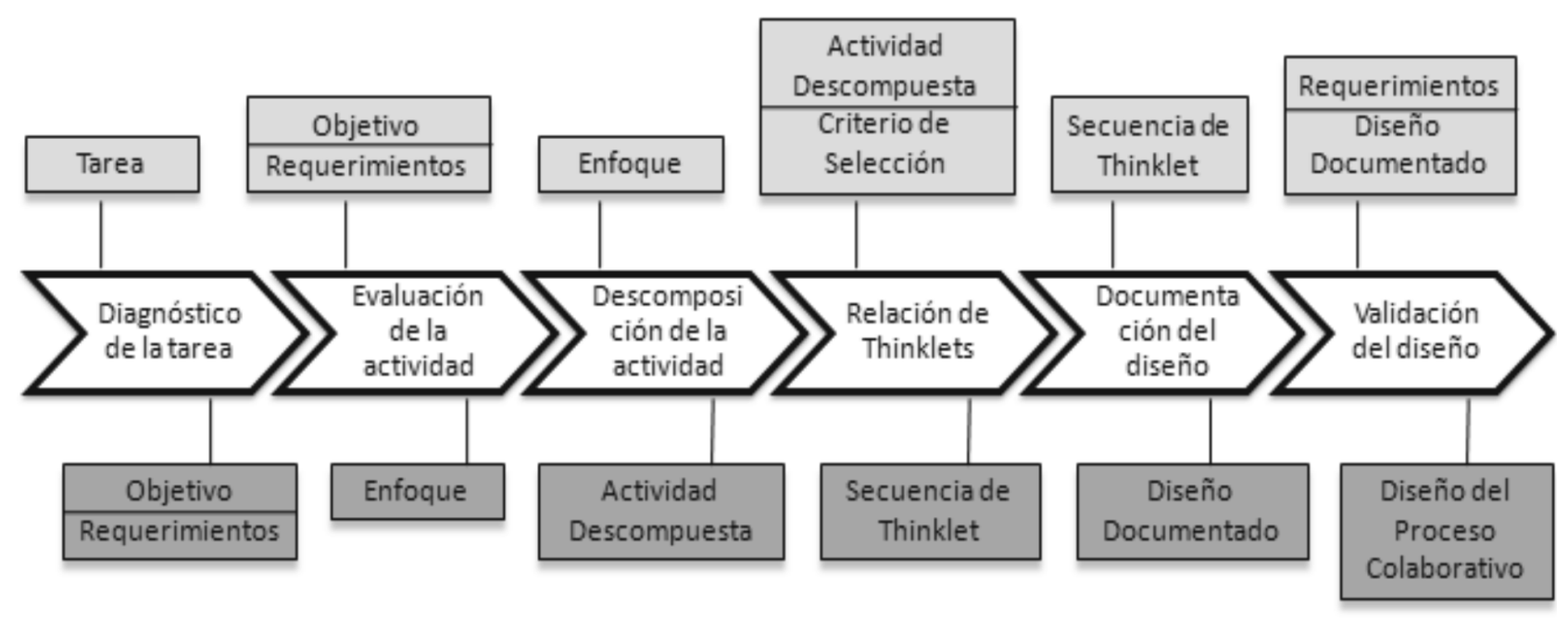

Figura 1. Metodología para el desarrollo de procesos colaborativos

Fuente: Kolfschoten et al [13]. 


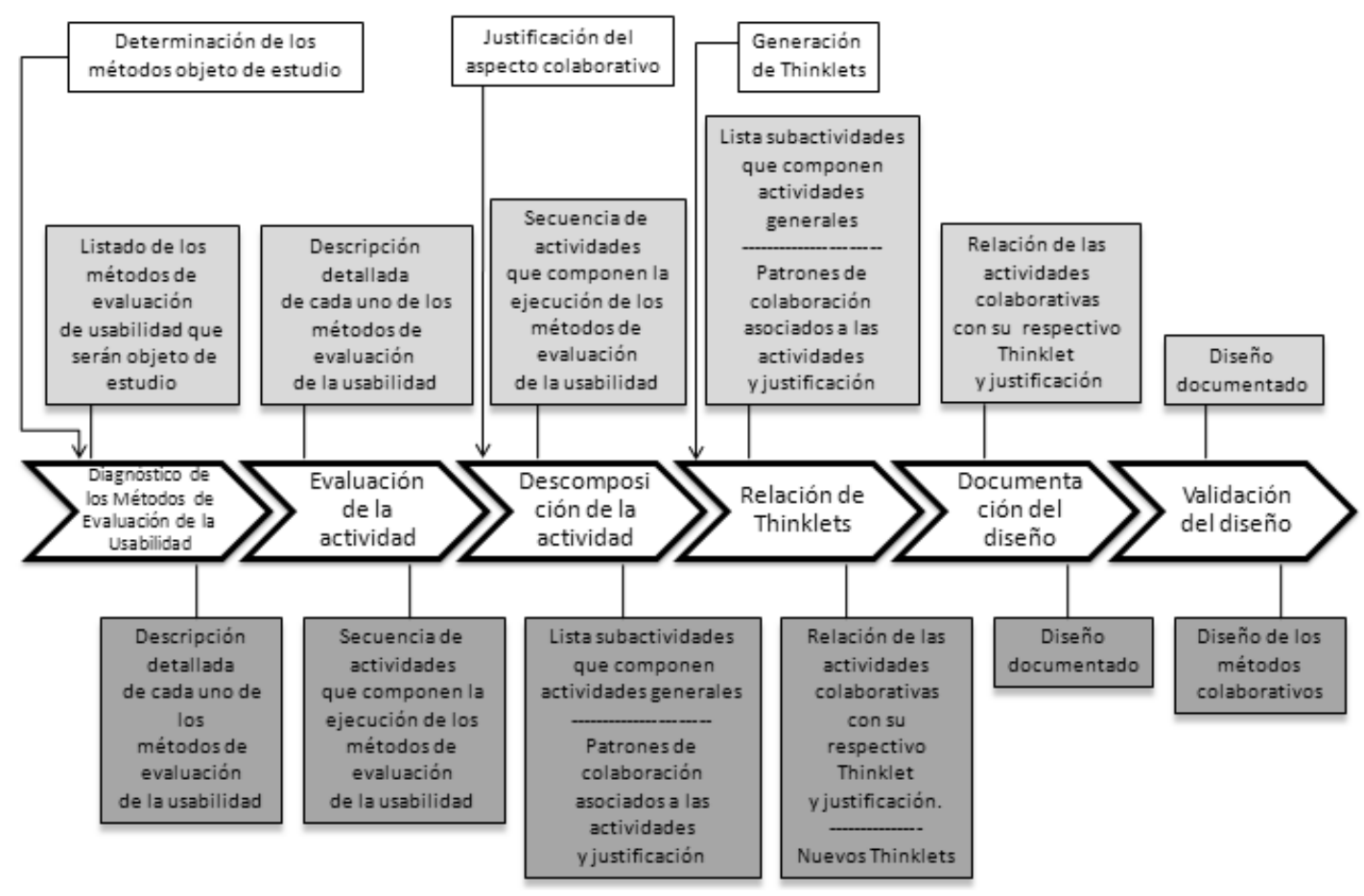

Figura 2. Metodología para el Desarrollo de Métodos de Evaluación de Usabilidad Colaborativos.

Fuente: Solano et al [12]

La primera fase que se denomina "Diagnóstico de la tarea", pasa a ser "Diagnóstico de los métodos de evaluación de usabilidad", y de acuerdo con lo anterior, se adicionaron las siguientes fases al proceso: Determinación de los métodos objeto de estudio, Justificación del aspecto colaborativo y Generación de nuevos thinklets (figura 2). En las figuras 1 y 2 , las cajas en una tonalidad gris clara de la parte superior, son las entradas que se requieren en cada fase y las cajas en una tonalidad gris oscura de la parte inferior, describen la salida de cada fase. En la figura 2 las cajas en blanco de la parte superior, corresponden a las fases adicionales propuestas en la metodología. En la siguiente sección, se presenta de forma general información relacionada con las fases que conforman la metodología.

\subsection{EJECUCIÓN DE LA METODOLOGÍA PARA EL DISEÑO DE MÉTODOS DE EVALUACIÓN DE USABILIDAD COLABORATIVOS}

La primera fase de la metodología (fase adicional), planteada para generar métodos de evaluación de usabilidad colaborativos, consiste en determinar los métodos objeto de estudio. Mediante una encuesta realizada a un grupo de expertos en usabilidad, se identificaron los métodos más utilizados en la actualidad y corresponden a la categoría de indagación, que son: Observación de Campo, Entrevista y Grupo de Discusión Dirigida. El grupo de encuestados estuvo conformado por: 
- $\quad$ Toni Granollers, experto en usabilidad y diseño centrado en el usuario de la Universidad de Lleida, España.

- Martha González, experta en usabilidad de la Universidad de Lleida, España.

- María Lili Villegas de la Universidad del Quindío, Colombia.

- William Giraldo de la Universidad del Quindío, Colombia.

- Mauricio Candamil, Comunicador Social y Web Máster del portal Web de la Universidad del Cauca, Colombia.

- July Jiménez de la Universidad del Cauca, Colombia.

En el presente artículo, el método de la Entrevista será objeto de estudio para incluir aspectos de trabajo colaborativo de los procesos que los conforman. A continuación, para este método se presenta de forma general, los resultados obtenidos en la realización de cada fase de la metodología.

\subsubsection{Fase: Diagnóstico de los métodos de evaluación de la usabilidad}

En esta fase, se realiza una descripción del método objeto de estudio, en la cual se incluyen los entregables, requerimientos y demás información relevante del método. En la tabla 1, se presenta la descripción del método de la Entrevista.

Tabla 1. Descripción del método de la Entrevista.

\section{Descripción del método}

\section{Entregables}

- Documento con información de la percepción de los usuarios frente a las funcionalidades del prototipo o sistema que se va a desarrollar o a rediseñar.

- Listado de problemas de usabilidad presentes en las funcionalidades del prototipo o sistema que se va a desarrollar o a rediseñar.

- Recomendaciones para solucionar los problemas de usabilidad identificados en el sistema.

Requerimientos: Prototipos o sistema final.

Recursos: Grabadora, Cámara de vídeo (opcional), Bloc de notas.

Etapa dentro del proceso de desarrollo: Este método puede ser utilizado en cualquier etapa del ciclo de vida dentro del proceso de desarrollo del sistema y al final del proceso de desarrollo, para evaluar el nivel de satisfacción del grupo de usuarios con el sistema [14].

Equipo de trabajo y roles: Se sugiere que el equipo de trabajo responsable de la ejecución del método, esté conformado por:

- Entrevistadores expertos: son las personas encargadas de ejecutar el método y analizar la información recolectada. Además, son quienes tienen el conocimiento y experiencia en la evaluación de usabilidad de software.

- Entrevistados: son los usuarios potenciales del sistema.

- Representante de la organización: persona de la organización que tiene conocimiento acerca del sistema por evaluar y estará presente durante la ejecución del método. 


\subsubsection{Fase: Evaluación de la actividad}

En esta fase, se identifican las actividades generales que componen el método objeto de estudio y se determina la secuencia entre ellas. En la tabla 2, se listan las actividades generales que componen el método de la Entrevista.

Tabla 2. Actividades generales del método de la Entrevista

\begin{tabular}{|r|l|}
\hline \multicolumn{1}{|c|}{$\mathbf{N}^{\mathbf{0}}$} & \multicolumn{1}{c|}{ Actividades } \\
\hline 1 & Determinar cuál es el sistema por evaluar. \\
\hline 2 & $\begin{array}{l}\text { Determinar las funcionalidades del sistema sobre las cuales se desea obtener informa- } \\
\text { ción. }\end{array}$ \\
\hline 3 & Decidir el orden de las funcionalidades. \\
\hline 4 & $\begin{array}{l}\text { Preparar el listado de preguntas y su orden correspondiente a las funcionalidades pre- } \\
\text { definidas para entrevistar a los usuarios del sistema. }\end{array}$ \\
\hline 5 & Determinar el tiempo límite de duración de la entrevista. \\
\hline 6 & Seleccionar los usuarios del sistema que serán entrevistados. \\
\hline 7 & Decidir cómo se va a registrar la respuesta del entrevistado. \\
\hline 8 & Elegir el lugar donde se va a realizar la entrevista. \\
\hline 9 & Desarrollo de la entrevista a los usuarios del sistema. \\
\hline 10 & Análisis de los datos obtenidos y conclusiones. \\
\hline
\end{tabular}

\subsubsection{Fase: Descomposición de la actividad}

En esta fase, se especifican las subactividades que componen cada actividad general identificada en la fase anterior. Luego de obtener las subactividades, se definen cuáles se realizarían de forma colaborativa, y a cada una de ellas se le asocia un patrón de colaboración.

Para determinar el conjunto de subactividades que conforman el método de la Entrevista, y cuáles requieren trabajo colaborativo, se realizó una encuesta a un grupo de expertos en usabilidad (conformado por las mismas personas que participaron en la encuesta mencionada anteriormente), con el objetivo de identificar las subactividades que requieren trabajo colaborativo para su ejecución. Por ejemplo: en la tabla 3, se listan las subactividades que componen la actividad general 10: Análisis de los datos obtenidos y conclusiones.

Tabla 3. Descomposición de la actividad general 10.

\begin{tabular}{|c|l|c|}
\hline $\mathbf{N}^{\mathbf{0}}$ & \multicolumn{1}{|c|}{ Subactividades } & Colaborativa \\
\hline 10.1 & $\begin{array}{l}\text { Analizar el contenido del documento de las entrevistas para obtener } \\
\text { información de la percepción de los usuarios frente a las funcionalidades } \\
\text { del sistema. }\end{array}$ & SI \\
\hline 10.2 & $\begin{array}{l}\text { Identificar los problemas de usabilidad presentes en las funcionalidades del } \\
\text { sistema, a partir del análisis realizado. }\end{array}$ & $\mathrm{SI}$ \\
\hline 10.3 & Generar recomendaciones para solucionar los problemas de usabilidad. & $\mathrm{SI}$ \\
\hline 10.4 & Plantear conclusiones. & \\
\hline \multicolumn{2}{|l|}{ Participantes: Representante de la organización y entrevistadores expertos } & \\
\hline
\end{tabular}


Respecto de la fase adicional: Justificación del aspecto colaborativo. Para determinar cuáles subactividades requieren trabajo colaborativo para su ejecución, es fundamental tener en cuenta los siguientes criterios [12]:

- La ejecución de la actividad y el plan de trabajo requieren incluir varias personas que pueden tener diferentes roles.

- Se requiere contar con personas que tienen un alto grado de experticia en un área específica de conocimiento, para ejecutar las actividades.

- Es necesario compartir conocimiento, recursos e información con otras personas.

- $\quad$ Es necesario tener en cuenta los diferentes aportes, opiniones y puntos de vista de otros integrantes del grupo que está ejecutando la actividad.

Posterior a la identificación de las subactividades que requieren trabajo colaborativo, se asocian patrones de colaboración a dichas subactividades. Por ejemplo, en la tabla 4 , se presentan los resultados de asociar patrones de colaboración a la subactividad colaborativa 10.1: Analizar el contenido del documento de las entrevistas para obtener información de la percepción de los usuarios frente a las funcionalidades del sistema.

Tabla 4. Asociación de patrones a la subactividad 10.1.

Subactividad: Analizar el contenido del documento de las entrevistas para obtener información de la percepción de los usuarios frente a las funcionalidades del sistema.

\section{Actividades relacionadas: 10.2}

\section{Entradas:}

- Documento de las entrevistas diligenciado, el cual contiene el listado ordenado de preguntas por cada funcionalidad, con sus respectivas respuestas. Adicionalmente presenta información como: día, fecha y hora de las entrevistas y nombres de los entrevistados.

- Listado de funcionalidades sobre las cuales se desea obtener información.

Resultados esperados: Información de la percepción de los usuarios frente a las funcionalidades del prototipo o sistema que se va a desarrollar o rediseñar.

Participantes: Entrevistadores expertos y representante de la organización.

\begin{tabular}{|l|l|}
\hline Patrones & Justificación \\
\hline Reducción & $\begin{array}{l}\text { Los entrevistadores pretenden reducir, interpretar y sistematizar la información } \\
\text { del documento de las entrevistas. }\end{array}$ \\
\hline Evaluación & $\begin{array}{l}\text { Los entrevistadores y el representante de la organización comparten sus } \\
\text { opiniones para comprender mejor el análisis realizado al documento de las } \\
\text { entrevistas. }\end{array}$ \\
\hline
\end{tabular}




\subsubsection{Fase: Relación de Thinklets}

Luego de asignar los patrones de colaboración a las subactividades colaborativas, se requiere asociar a cada patrón seleccionado un thinklet que se adecúe a los procesos que conforman dicha subactividad. Los thinklets identificados deben adecuarse a los recursos, al grupo y hasta las propias habilidades de las personas involucradas en la ejecución de los procesos colaborativos [9]. En la tabla 5, se presentan los resultados obtenidos de la relación de thinklets a la subactividad 10.1: Analizar el contenido del documento de las entrevistas para obtener información de la percepción de los usuarios frente a las funcionalidades del sistema.

Tabla 5. Relación de Thinklets de la subactividad 10.1.

\begin{tabular}{|c|c|l|}
\hline \multicolumn{3}{|c|}{$\begin{array}{l}\text { Subactividad: Analizar el contenido del documento de las entrevistas para obtener información de la } \\
\text { percepción de los usuarios frente a las funcionalidades del sistema }\end{array}$} \\
\hline Patrones & Thinklet & Justificación de selección del thinklet \\
\hline Reducción & $\begin{array}{c}\text { AnalysisCo } \\
\text { ntent }\end{array}$ & $\begin{array}{l}\text { Este thinklet permite realizar conclusiones a partir del análisis de las } \\
\text { respuestas a preguntas abiertas. } \\
\text { Los pasos de este Thinklet se ajustan de forma adecuada al proceso } \\
\text { que conforma la actividad. }\end{array}$ \\
\hline Evaluación & StrawPoll & $\begin{array}{l}\text { Este thinklet permite a los entrevistadores y representante de la } \\
\text { organización evaluar el conjunto de conclusiones obtenidas con el } \\
\text { thinklet Analysis Content. } \\
\text { Los pasos que ofrece este thinklet se ajustan de forma adecuada al } \\
\text { proceso que conforma la actividad. }\end{array}$ \\
\hline
\end{tabular}

Respecto de la fase adicional: Generación de thinklets, se propusieron los thinklets: Discussion Circle y Analysis Content. La información relacionada con estos thinklets se presenta en las secciones 3.2.1 y 3.2.2.

\subsubsection{Fase: Documentación del diseño}

A partir de la información obtenida en las fases anteriores, se genera la documentación definitiva del método colaborativo objeto de estudio. Para tal fin, la Ingeniería de Colaboración ha definido los siguientes elementos [13]:

Descripción del Proceso: presenta información general relacionada con el proceso colaborativo diseñado. En la tabla 6 , se presenta la descripción del proceso de la actividad general 10: Análisis de los datos obtenidos y conclusiones, que está conformada por subactividades colaborativas. 
Tabla 6. Descripción del proceso de la actividad general 10

\section{Descripción del proceso}

\section{Objetivos}

Analizar el contenido del documento de las entrevistas para obtener información de la percepción de los usuarios frente a las funcionalidades del sistema.

Identificar problemas de usabilidad presentes en las funcionalidades objeto de estudio.

Generar recomendaciones para solucionar a los problemas de usabilidad identificados.

Realizar conclusiones del análisis realizado a la información recolectada.

\section{Entregables}

- Información de la percepción de los usuarios frente a las funcionalidades del sistema.

- Listado de problemas de usabilidad identificados en las funcionalidades del sistema objeto de estudio.

- Recomendaciones para solucionar los problemas de usabilidad identificados en las funcionalidades del sistema.

- Conclusiones del análisis realizado a la información recolectada.

\section{Requerimientos}

- Listado de funcionalidades sobre las cuales se desea obtener información.

- Documento de las entrevistas diligenciado que contiene el listado ordenado de preguntas por cada funcionalidad con sus respectivas respuestas. Adicionalmente, presenta información como: fecha, hora de las entrevistas y nombres de los entrevistados.

- Criterios para organizar los problemas de usabilidad y las recomendaciones, definidos por el representante de la organización según las necesidades de la organización.

\section{Visión General}

Los responsables de ejecutar esta actividad son los entrevistadores expertos y el representante de la organización.

Los entrevistadores analizan el contenido del documento de las entrevistas para obtener información de la percepción de los usuarios frente a las funcionalidades objeto de estudio. A partir del análisis realizado al documento de las entrevistas, los entrevistadores identifican los problemas de usabilidad presentes en dichas funcionalidades y hacen recomendaciones para solucionar cada problema. Por último, los entrevistadores en compañía del representante de la organización plantean algunas conclusiones a partir del análisis de la información recolectada.

Agenda Detallada: presenta de manera clara y detallada la información relacionada con cada actividad que hace parte del proceso diseñado. La agenda detallada contiene información como: número, actividad, entregable, thinklet y patrón de colaboración, proceso colaborativo, tiempo estimado y participantes de cada actividad que conforma el método objeto de estudio. La información del proceso colaborativo es una descripción detallada de los pasos que se requieren para ejecutar la actividad (estos corresponden a los pasos propuestos en el thinklet asociado, con sus respectivas adaptaciones). En la tabla 7, se presenta una porción de la agenda detallada con las subactividades que conforman la actividad general 10: Análisis de los datos obtenidos y conclusiones. En la porción de la agenda detallada no se incluye información relacionada con el proceso colaborativo. 
Tabla 7. Porción de Agenda detallada con las subactividades que componen la actividad 10.

\begin{tabular}{|c|c|c|c|c|c|}
\hline $\mathbf{N}^{\circ}$ & Actividad & Entregable & Thinklet y Patrón & $\begin{array}{c}\text { Tiempo } \\
\text { estimado } \\
\text { (min) }\end{array}$ & Participantes \\
\hline \multicolumn{6}{|c|}{ ACTIVIDAD 9. DESARROLLO DE LA ENTREVISTAA LOS USUARIOS DEL SISTEMA } \\
\hline \multicolumn{6}{|c|}{ ACTIVIDAD 10: ANÁLISIS DE LOS DATOS OBTENIDOS Y CONCLUSIONES } \\
\hline \multicolumn{6}{|c|}{$\begin{array}{l}\text { Subactividad 10.1: Analizar el contenido del documento de las entrevistas para obtener información } \\
\text { de la percepción de los usuarios frente a las funcionalidades del sistema. }\end{array}$} \\
\hline 10.1.1 & $\begin{array}{c}\text { Analizar el } \\
\text { contenido del } \\
\text { documento de } \\
\text { las entrevistas. }\end{array}$ & $\begin{array}{l}\text { Información de la } \\
\text { percepción de los } \\
\text { usuarios frente a las } \\
\text { funcionalidades } \\
\text { objeto de estudio. }\end{array}$ & $\begin{array}{l}\text { AnalysisContent } \\
\quad \text { (reducción) }\end{array}$ & 180 & $\begin{array}{l}\text { Entrevistadores } \\
\text { Expertos }\end{array}$ \\
\hline 10.1 .2 & $\begin{array}{l}\text { Compartir } \\
\text { opiniones para } \\
\text { comprender } \\
\text { mejor el análisis } \\
\text { realizado al } \\
\text { documento de } \\
\text { las entrevistas }\end{array}$ & $\begin{array}{l}\text { Documento definitivo } \\
\text { con conclusiones de } \\
\text { la percepción de los } \\
\text { usuarios frente a las } \\
\text { funcionalidades } \\
\text { objeto de estudio. }\end{array}$ & $\begin{array}{l}\text { StrawPoll } \\
\text { (evaluación) }\end{array}$ & 20 & $\begin{array}{c}\text { Representante } \\
\text { de la } \\
\text { Organización, } \\
\text { Entrevistadores } \\
\text { Expertos }\end{array}$ \\
\hline 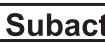 & dad 10.2: & & & & \\
\hline
\end{tabular}

La información relacionada al Thinklet Analysis Content se presenta en la sección 3.2.2, el Thinklet Straw Poll ha sido generado a partir de investigaciones en Ingeniería de Colaboración [15].

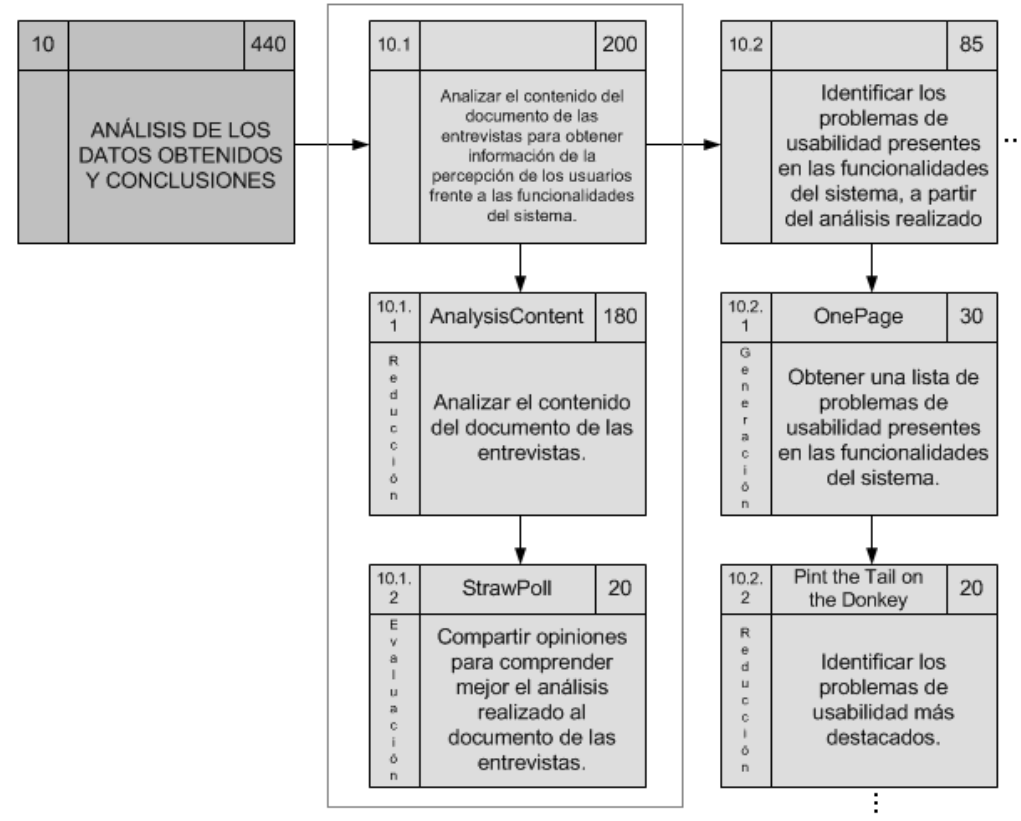

Figura 3. Porción de MFP de la actividad general 10. 
Modelo de Facilitación del Proceso (MFP): es utilizado para mostrar el flujo del proceso y los elementos tales como thinklet, identificador correspondiente a la agenda detallada, tiempo estimado de duración, patrón de colaboración y descripción, relacionados con cada actividad que conforma el proceso. En la figura 3, se presenta una porción del MFP con las subactividades que conforman la actividad general 10: Análisis de los datos obtenidos y conclusiones.

\subsubsection{Fase: Validación del diseño}

Por último, se debe validar el diseño del método colaborativo objeto de estudio; para tal fin, la metodología ofrece las siguientes formas de validación [13]:

- Prueba piloto: consiste en ejecutar el método colaborativo diseñado para evaluar la efectividad del proceso. El objetivo es verificar si la ejecución de los métodos se puede llevar a cabo en el tiempo estimado y con los recursos definidos.

- Recorrido: consiste en realizar la evaluación del método colaborativo con algunos de los participantes en la ejecución del mismo, para identificar falencias y dificultades durante la ejecución.

- $\quad$ Simulación: el equipo de trabajo que diseñó el método colaborativo, responde a una serie de preguntas; esta validación prueba la lógica del diseño y si a cada paso se le creará el entregable requerido. Las respuestas generadas servirán de referente para realizar las mejoras respectivas.

- Revisión: consiste en generar discusión sobre los diseños realizados entre el equipo de trabajo y los potenciales usuarios que ejecutarán el método colaborativo, para ayudar a identificar partes del diseño ineficientes.

Para la validación preliminar del método colaborativo de la Entrevista, se utilizaron dos formas de validación de las establecidas por la metodología, las cuales son: Simulación y Prueba Piloto. Estas formas de validación permitieron probar la lógica del diseño, comprobar que a cada paso se le crean los entregables requeridos, identificar problemas en el diseño, verificar si la ejecución de las actividades colaborativas se puede llevar a cabo en el tiempo estimado y con los recursos definidos y de esta manera, mejorar el diseño del método.

\section{GENERACIÓN DE THINKLETS}

\subsection{IMPORTANCIA DEL THINKLET EN EL DISEÑO DE MÉTODOS COLABORATIVOS PARA EVALUAR USABILIDAD DE SOFTWARE}

Los thinklets pueden usarse para construir grupos de procesos que conforman métodos de evaluación de usabilidad de software; son elementos creados para ser utilizados por 
personas con poca experiencia, ya que son fácilmente aprendidos, recordados y pueden adaptarse a un diseño de proceso [16]. En el contexto de los métodos de evaluación de usabilidad, el uso de thinklets resulta conveniente por que:

- Soportan el diseño de procesos colaborativos.

- $\quad$ Sirven como lenguaje común entre los diseñadores de procesos colaborativos.

- Sirven como punto de partida para la ejecución de un proceso colaborativo.

- $\quad$ Sirven como instrumento de investigación para comparar la ejecución de diferentes procesos colaborativos.

Una de las grandes ventajas de los thinklets es que los diseñadores de procesos colaborativos pueden emplearlos para escoger soluciones conocidas y no invertir esfuerzos en inventar y probar nuevas. Esto puede reducir tanto el esfuerzo como el riesgo, durante el desarrollo de procesos colaborativos [15]. Con el propósito de contribuir en estos aspectos, en la siguiente sección se presentan algunos thinklets, generados por el grupo de trabajo, que intentan facilitar el desarrollo de procesos colaborativos.

\subsection{THINKLETS GENERADOS}

El grupo de trabajo ha generado nuevos thinklets, que se consideran útiles a la Ingeniería de Colaboración, estos son: DiscussionCircle, DiscussionChips y AnalysisContent. Los thinklets: DiscussionCircle y DiscussionChips, se basan en las técnicas de aprendizaje colaborativo: Inside-Outside Circle y Talking Chips [17], respectivamente. Estas técnicas permiten que un grupo de personas hablen de un tema común, con la particularidad de que se garantiza igual participación de las personas. El Thinklet AnalysisContent se basa en la técnica de análisis de contenido [18], que es de gran utilidad para analizar grandes cantidades de información de una manera objetiva, sistemática y cuantitativa. A continuación, se presenta la descripción de los thinklets DiscussionCircle y AnalysisContent.

\subsubsection{Thinklet: DiscussionCircle}

Este Thinklet se basa en la técnica de aprendizaje colaborativo Inside-Outside Circle, que "permite a un grupo de personas intercambiar ideas con la participación de todos" [17]. El proceso de la técnica fue adecuado de tal manera que pudiera ser utilizado en la ejecución de métodos colaborativos de evaluación de usabilidad. En la tabla 8, se presenta la descripción del thinklet DiscussionCircle. 
Tabla 8. Thinklet: DiscussionCircle

\section{Discussion Circle}

\section{Escoger este Thinklet...}

- Cuando sea necesario realizar una discusión sobre un tema particular, entre un grupo de no menos de seis y no más a diez personas.

-Cuando se necesita que los miembros del grupo generen discusión con base en información generada por otro miembro del grupo.

-Cuando en una discusión se quiere lograr igual participación del grupo.

\section{No escoger este Thinklet...}

- Cuando se tengan menos de seis personas en el grupo de trabajo.

- Cuando el grupo de trabajo esté conformado por más de diez personas, el tiempo demorado en lograr el objetivo de la actividad es muy largo, por que los pasos que conforman el Thinklet son un poco extensos.

- Si no se cuenta con una persona que ejerza el rol de moderador.

Información general: Este thinklet permite a un grupo de trabajo desarrollar de forma adecuada, una discusión sobre un tema en particular y logra igual participación de todos sus integrantes. Los integrantes del grupo realizan la discusión de un tema particular en parejas durante un tiempo determinado, se rotan las parejas para iniciar una nueva discusión del mismo tema, se continúa rotándo el número de veces deseado o hasta que el tiempo lo permita. Después se hace una discusión con todos los integrantes del grupo, aquí se discute nuevamente el tema. Esta actividad se realiza con cada tema por discutir.

Entradas: Un conjunto de temas que serán discutidos por el grupo.

Salidas: Conjunto de ideas, opiniones, preferencias y gustos de los usuarios respecto de los temas discutidos.

\section{Pasos}

1. El moderador selecciona un tema para discutir.

2. El moderador inicia la discusión con la primera pregunta. Los integrantes del grupo se organizan en parejas. Se discute la pregunta, siguiendo los siguientes pasos: 1) las parejas forman un círculo, 2) las personas del círculo exterior intercambian ideas con las personas que tienen enfrente (pertenecientes al círculo interior), durante un tiempo máximo de tres minutos.

3. Después, las personas del círculo exterior rotan una posición de tal forma que ahora observan otra persona del círculo interior y realizan de nuevo una discusión.

4. Una vez las personas del círculo exterior hayan discutido con todas las personas del círculo interior, se realiza una discusión entre todos los integrantes del grupo de trabajo, en la cual cada uno da sus aportes.

5. Los pasos 2 a 4 se realizan para cada una de las preguntas que conforman el tema de discusión.

6. Continuar moderando la actividad hasta que se llegue al límite de tiempo previamente determinado o hasta que ya no se tengan más temas para discutir por parte de los participantes.

\section{Observaciones}

Para la realización de este thinklet, se recomienda contar con una persona que ejerza el rol de moderador, para guiar al grupo, mantener la atención de los integrantes en los temas de interés, permitir la libre circulación de ideas y comentarios, además de garantizar que todos los participantes contribuyan en la discusión y evitar que opine un solo participante. 


\section{Discussion Circle}

\section{Historia Exitosa}

Este thinklet se ha utilizado para generar una discusión entre un grupo de usuarios del sitio Web del convenio entre la Universidad del Cauca y el programa Computadores para Educar (CPE). La discusión se realizó con el objetivo de obtener información sobre las necesidades, preferencias y gustos de los usuarios respecto del sitio Web. La ejecución de este thinklet permitió a los integrantes del grupo de trabajo, obtener gran cantidad de información respecto de los temas que se estaban investigando.

\section{Explicación del Nombre}

El nombre DiscussionCircle surgió por que en la realización de este thinklet, todos los integrantes del grupo se mueven en círculo mientras discuten un tema de interés.

\subsubsection{Thinklet: Analysis Content}

El Thinklet AnalysisContent se basa en la técnica de análisis de contenido que es de gran utilidad para la reducción de datos. En la tabla 9, se presenta la descripción del Thinklet AnalysisContent.

Tabla 9. Thinklet: AnalysisContent

\section{AnalysisContent}

\section{Escoger este Thinklet...}

Cuando sea necesario reducir, interpretar y sistematizar cualquier tipo de información acumulada (por ejemplo: documentos escritos, trascripción de entrevistas, grabaciones, entre otros).

Para realizar deducciones o inferencias a partir del análisis detallado de las respuestas a preguntas abiertas.

\section{No escoger este Thinklet...}

Cuando los integrantes del grupo cuenten con poco tiempo para la ejecución, por que los pasos que conforman el Thinklet son extensos.

\section{Información general}

AnalysisContent es un thinklet que permite a los integrantes del grupo, analizar grandes cantidades de información de una manera objetiva, sistemática y cuantitativa. Se basa en el análisis de contenido que "es una técnica de investigación destinada a formular, a partir de ciertos datos, inferencias reproducibles y válidas que pueden aplicarse a su contexto" [18].

Entradas: Conjunto de fuentes de datos, por ejemplo: textos escritos, entrevistas, entre otros.

Salidas: Análisis objetivo y detallado de las fuentes de datos.

\section{Pasos}

1. Pedir a los integrantes del grupo que identifiquen la (s) fuente (s) de datos (por ejemplo: respuestas a preguntas abiertas de una entrevista, grabaciones).

2. Los participantes deben identificar las variables sobre las cuales se desea obtener información (por ejemplo: facilidad de aprendizaje para los usuarios de la funcionalidad $X$ de un aplicación software $Y$ ). 


\section{AnalysisContent}

3. Los integrantes del grupo identifican las diferentes preguntas correspondientes a cada variable objeto de estudio.

4. Los miembros del grupo identifican las diferentes alternativas de respuesta a cada pregunta.

5. Codificar las respuestas de las preguntas, para lo cual se debe realizar los siguientes pasos:

- Cada pregunta tendrá más de una posible respuesta, por lo cual a cada posible respuesta se le debe asignar un valor numérico (los valores numéricos asignados deben seguir una secuencia lógica), para poder realizar después, su análisis estadístico.

- Se debe asignar otros valores numéricos a los casos que no se considere posible asignarles un valor de los establecidos.

6. Elaborar el libro de códigos [19], que tiene la siguiente información:

- Tabla con cuatro columnas: en la primera columna se ubican los nombres de las variables objeto de estudio; en la segunda, las preguntas que pretende medir la variable respectiva; en la tercera columna, las posibles respuestas asociadas a cada pregunta; y en la cuarta columna, el valor numérico asignado a cada respuesta.

- Descripción de cada una de las variables que se desean investigar.

- Descripción de la forma como se debe llenar la hoja de codificación, sabiendo qué valor numérico corresponde a las posibles respuestas de la pregunta analizada.

- Descripción sobre la forma como se concluye el diligenciamiento de la hoja de codificación. Elaborar la hoja de codificación. Los integrantes del grupo deben diseñar una hoja en donde se realiza la codificación de las preguntas correspondientes a cada variable objeto de estudio. La estructura de la hoja de codificación con sus correspondientes variables y casillas para el registro de datos, debe ser fácil de llenar, de manera que cada integrante del grupo aprenda rápidamente, sin tener que recurrir más de una vez al libro de códigos.

7. Los integrantes del grupo diligencian la hoja de codificación, codificando la información de la fuente de datos.

8. Motivar a los participantes a revisar la información registrada en la hoja de codificación, con el fin de encontrar posibles errores (en la asociación de los valores numéricos con las posibles respuestas de una pregunta), corregirlos y así poder mejorar la calidad de la información codificada.

9. Los integrantes del grupo analizan los resultados de la hoja de codificación (para esto se pueden utilizar herramientas software), y rhacen conclusiones sobre dicho análisis.

10.Continuar moderando la actividad hasta que se llegue al límite de tiempo previamente determinado o hasta que ya no se tengan más conclusiones por parte de los participantes.

\section{Observaciones}

Los datos una vez recolectados en las hojas de codificación, están en condiciones de ser sistematizados para su procesamiento y análisis estadístico. Se recomienda para el análisis de contenido, que los datos recolectados estén en archivos informáticos que pueden ser importados a programas estadísticos de tratamiento de datos (la utilización de herramientas software conlleva a un análisis estadístico más eficaz).

\section{Historia Exitosa}

Se ha utilizado este thinklet para analizar el contenido de entrevistas, con el fin de obtener información de la percepción de los usuarios frente a las funcionalidades que ofrece el sitio Web del convenio entre la Universidad del Cauca y el programa Computadores para Educar 


\section{AnalysisContent}

(CPE). La ejecución de este thinklet permitió a los integrantes del grupo de trabajo, obtener información confiable y objetiva sobre la satisfacción, gustos, opiniones y preferencias de los usuarios. El grupo de trabajo empleó tres horas en ejecutar el thinklet.

\section{Explicación del Nombre}

El nombre AnalysisContent se debe a que el thinklet se basa en la técnica de análisis de contenido.

\section{CONCLUSIONES Y TRABAJO FUTURO}

Las empresas desarrolladoras de software se podrían beneficiar con los métodos colaborativos para evaluar la usabilidad de software obtenidos a partir del desarrollo de la metodología propuesta, ya que al integrar varias personas de diferentes áreas de experticia en el proceso de evaluación de usabilidad de los productos, comparten conocimiento y recursos, garantizando así la obtención de productos software más usables para los usuarios finales.

Debido a que en los métodos de evaluación de usabilidad se intenta fomentar el trabajo colaborativo entre los integrantes de un grupo, se contribuye significativamente para que durante la realización de las actividades colaborativas se generen resultados más adecuados al respecto del trabajo grupal. Al trabajar de forma colaborativa, se generan para los integrantes del grupo ventajas como: permitir entender mejor un problema, facilitar la detección de errores, dar más alternativas de solución a un problema, acceder a un mayor volumen de información útil, entre otras. Esta forma de apoyar los procesos de evaluación de la usabilidad puede mejorar notablemente la calidad del producto y el método de una manera innovadora.

En cada actividad colaborativa, se intenta fortalecer la colaboración entre los diferentes miembros del grupo, es decir, se intenta promover la comunicación, la coordinación y la negociación, con el fin de aumentar la productividad. La colaboración permite a los miembros del grupo, unir esfuerzos intelectuales para buscar un objetivo común que en este caso particular, es lograr evaluar la usabilidad de productos software de una manera más acertada.

La generación de nuevos thinklets es muy pertinente para la Ingeniería de Colaboración, debido a que los existen no son suficientes; algunos de estos, no se adaptan a las necesidades específicas de un proyecto y se incrementa el número de opciones para seleccionar en el momento de asociar thinklets a una actividad colaborativa. Los nuevos thinklets: DiscussionCircle y AnalysisContent, integran aspectos como: el análisis de contenido y técnicas que permiten la discusión de temas comunes, garantizando la participación de todas las personas que conforman el grupo. 
Sería un gran aporte diseñar de forma colaborativa todos los métodos de evaluación de usabilidad, pertenecientes a las categorías de inspección, indagación y prueba. Es conveniente continuar mejorando el diseño del método colaborativo de la Entrevista y generar nuevos thinklets, debido a que algunos de estos no se adaptan a las necesidades específicas de un proyecto. Además, es adecuado continuar mejorando los thinklets generados por el grupo de trabajo.

\section{AGRADECIMIENTOS}

Este trabajo ha sido parcialmente financiado por los proyectos: Implementación de un Framework para la evaluación de la Usabilidad de aplicaciones software soportado en la creación de un Colaboratorio de usabilidad, código 111345221103 de COLCIENCIAS; Lineamientos de usabilidad para el diseño y desarrollo de servicios educativos para Televisión Digital Interactiva, código 2713 de la Vicerrectoría de Investigaciones de la Universidad del Cauca (Colombia), y el proyecto ST-CAV: Servicios de T-learning para el soporte de una Comunidad Académica Virtual, Código 110348925425, financiado por COLCIENCIAS y el SENA.

\section{REFERENCIAS BIBLIOGRÁFICAS}

[1] Lucero, M. (2004). Entre el Trabajo Colaborativo y el Aprendizaje Colaborativo; En: Revista Iberoamericana de Educación. Facultad de Ciencias Fisico-matemáticas y Naturales. ISSN: 1681-5653.

[2] ISO 9241-11 (1998). Ergonomic requirements for office work with visual display terminals.

[3] Lores, J., Granollers, T., Sergi, L. (2002). Introducción a la Interacción Persona Ordenador, Universidad de Lleida.

[4] Alzate, B. (2008). Evaluación de Interfaces Gráficas: ¿Qué, Cómo, Cuándo, Quién, Cuánto y a Qué Precio? Grupo de Investigación Centro Imagen GICI Escuela de Arquitectura y Diseño Universidad Pontificia Bolivariana, Medellín, Colombia.

[5] Marx, R. (1884). La "Neue Rheinische Zeitung. Organ der Demokratie"; Nueva Gaceta del Rin, Órgano de la Democracia.

[6] Bannon, L., Schmidt, K. (1989). CSCW: Four Characters in Search of a Context. In Proc. First European Conf. On CSCW, Gatwick, UK.

[7] Díaz, M. (2000). E-business: Tecnología de información y redes de negocios. Debates IESA. 
[8] Trabajo colaborativo. Consultada en marzo de 2011. En: http://www.ucla.edu.ve/ dac/investigaci\%F3n/compendium6/Tecnologia\%20de\%20trabajo\%20colaborativo. $\mathrm{htm}$

[9] Kolfschoten, G., Briggs, R., Vreede, G. (2006). Definitions in Collaboration Engineering; Proceedings of the 39 Hawaii International Conference on System Sciences. Delft University of Technology, University of Arizona.

[10] Chatterjee, S., Fuller, M., Sarker, S. (2007). An Ethical Design Theory for Thinkletbased Collaboration: Washington State University.

[11] Briggs, R., Vreede, G., Nunamaker, J., Tobey, D. (2001). Thinklets: Achieving Predictable, Repeatable Patterns of Group Interaction with Group Support Systems (GSS); Proceedings of the $34^{\text {th }}$ Hawaii International Conference on Systems Sciences. Delft University of Technology, University of Arizona.

[12] Solano, A., Parra, C., Collazos, C., Méndez, Y. (2010). Evaluación de Usabilidad de Software desde una Perspectiva Colaborativa; Conferencia Latinoamericada de Medios Audiovisuales en Red - LACNEM 2010.

[13] Kolfschoten, G., Vreede, G., Chakrapani, A., Koneri, P. (2006). The Collaboration Engineering Approach for Designing Collaboration Processe; Proceedings of the 39 Hawaii International Conference on System Sciences. Delft University of Technology, University of Arizona.

[14] Nielsen, J. (1993). Usability Engineering. AP Professional. Boston, USA.

[15] Kolfschoten, G., Briggs, R., Appelman, J., Vreede, G. (2004). Thinklets as Building Blocks for Collaboration Processes: A Further Concept. Lecture notes in computer science, Berlin, Springer Verlag.

[16] Vreede, G., Briggs, R. (2005). Collaboration Engineering: Designing Repeatable Processes for HighValue Co. Proceedings of the 38 Hawaii International Conference on System Sciences, Delft University of Technology, University of Arizona.

[17] Kagan, S. (1992). Cooperative Learning; San Juan Capistrano, CA: Kagan Cooperative Learning, USA.

[18] Krippendorf, K. (1990) Metodología de análisis de contenido: Teoría y práctica. Barcelona, Piados Ibérica, p.11.

[19] Piñuel, J. (2002). Epistemología, metodología y técnicas del análisis de contenido: Universidad Complutense de Madrid, España. 